\title{
Oligometastatic Prostate Adenocarcinoma. Clinical-Pathologic Study of a Histologically Under-Recognized Prostate Cancer
}

\author{
Claudia Manini ${ }^{1, \dagger}{ }^{\dagger}$ Alba González ${ }^{2,+}$, David Büchser ${ }^{2}$, Jorge García-Olaverri ${ }^{3}$, \\ Arantza Urresola ${ }^{4}$, Ana Ezquerro ${ }^{4}$, Iratxe Fernández ${ }^{5}$, Roberto Llarena ${ }^{3}$, Iñaki Zabalza ${ }^{6}$, \\ Rafael Pulido $7,8,9$ (), Arkaitz Carracedo ${ }^{8,9,10,11}{ }^{\circledR}$, Alfonso Gómez-Iturriaga ${ }^{2,7, *}$ \\ and José I. López ${ }^{7,12, *(1)}$ \\ 1 Department of Pathology, San Giovanni Bosco Hospital, 10154 Turin, Italy; claudiamaninicm@gmail.com \\ 2 Department of Radiation Oncology, Cruces University Hospital, 48903 Barakaldo, Spain; \\ albag1.91@gmail.com (A.G.); david.buechsergarcia@osakidetza.eus (D.B.) \\ 3 Department of Urology, Cruces University Hospital, 48903 Barakaldo, Spain; \\ jorge.garciaolaverrirodriguez@osakidetza.eus (J.G.-O.); roberto.llarenaibarguren@osakidetza.eus (R.L.) \\ 4 Department of Radiodiagnostics, Cruces University Hospital, 48903 Barakaldo, Spain; \\ aranzazu.urresolaolabarrieta@osakidetza.eus (A.U.); anaisabel.ezquerroimas@osakidetza.eus (A.E.) \\ 5 Department of Nuclear Medicine, Cruces University Hospital, 48903 Barakaldo, Spain; \\ iratxe.fernandez@osakidetza.eus \\ 6 Department of Pathology, Galdakao Hospital, 48960 Galdakao, Spain; inaki.zabalzaestevez@osakidetza.eus \\ 7 Biocruces-Bizkaia Health Research Institute, 48903 Barakaldo, Spain; rpulidomurillo@gmail.com \\ 8 Ikerbasque, The Basque Foundation for Science, 48009 Bilbao, Spain; acarracedo@cicbiogune.es \\ 9 Department of Cell Biology and Histology, Faculty of Medicine and Nursing, University of the Basque \\ Country (UPV/EHU), 48940 Leioa, Spain \\ 10 Center for Cooperative Research in Biosciences, Basque Research and Technology Alliance (BRTA), \\ 48160 Derio, Spain \\ 11 CIBERONC, 28046 Madrid, Spain \\ 12 Department of Pathology, Cruces University Hospital, 48903 Barakaldo, Spain \\ * Correspondence: agomeziturriaga@gmail.com (A.G.-I.); jilpath@gmail.com (J.I.L.); \\ Tel.: +34-94-600-6084 (J.I.L.) \\ + Equal contributors.
}

Received: 30 October 2020; Accepted: 2 December 2020; Published: 4 December 2020

\begin{abstract}
The clinical parameters and the histological and immunohistochemical findings of a prospective protocolized series of 27 prostate carcinoma patients with oligometastatic disease followed homogeneously were analyzed. Lymph nodes $(81.5 \%)$ and bones $(18.5 \%)$ were the only metastatic sites. Local control after metastatic directed treatment was achieved in $22(81.5 \%)$ patients. A total of $8(29.6 \%)$ patients developed castration-resistant prostate cancer. Seventeen $(63 \%)$ patients presented with non-organ confined disease. The Gleason index 8-10 was the most frequently observed (12 cases, $44.4 \%$ ) combined grade. Positive immunostainings were detected with androgen receptor $(100 \%)$, PGP $9.5(74 \%)$, ERG (40.7\%), chromogranin A (29.6\%), and synaptophysin (18.5\%) antibodies. The Ki- 67 index value $>5 \%$ was observed in $15 \%$ of the cases. L1CAM immunostaining was negative in all cases. Fisher exact test showed that successful local control of metastases was associated to mild inflammation, organ confined disease, $\mathrm{Ki}-67$ index $<5 \%$, and Gleason index $3+3$. A castration resistant status was associated with severe inflammation, atrophy, a Gleason index higher than $3+3$, Ki-67 index $\geq 5 \%$, and positive PGP 9.5, chromogranin A, and synaptophysin immunostainings. In conclusion, oligometastatic prostate adenocarcinoma does not have a specific clinical-pathologic profile. However, some histologic and immunohistochemical parameters of routine use may help with making therapeutic decisions.
\end{abstract}


Keywords: prostate cancer; oligometastatic disease; histopathology; inflammation; atrophy; gleason index; pathologic staging; immunohistochemistry; prognosis

\section{Introduction}

Prostate adenocarcinoma (PCa) is the most common neoplasm and a leading cause of mortality in the male population in Western countries, with more than 191,000 new cases and 33,000 deaths expected in the USA in 2020 [1]. The biology of this tumor is complex and sets forth very different clinical contexts that are not always easy to manage in practice.

A high percentage of PCa are detected in an organ-confined stage, and radical surgery or radiotherapy is the recommended treatment [2]. In this group of patients, however, the definition and management of the so-called clinically insignificant disease remains controversial [3], as well as the role of active surveillance $[4,5]$ and focal therapy [6] as alternative options.

On the other side of the spectrum, a significant subset of patients presents with a disseminated poly-metastatic status early in the clinical evolution, even at diagnosis. PCa in these patients encompasses high levels of intratumor heterogeneity and a wide spectrum of genomic alterations [7], with androgen-deprivation therapy as the main alternative with or without synchronous or metachronous chemotherapy or second-line antiandrogen regimes [8].

In between these two clinical contexts, a group of PCa patients is characterized by developing what it is called an oligometastatic status (OPCa), that is, a clinical situation in which a limited number of metastases, usually between one and five, appear during the course of the disease [9]. Since OPCa still has not been morphologically, immunohistochemically, or genomically characterized, this group of patients remains stratified simply under a clinical concept with unpredictable prognosis and treatment that has not yet been established. In this particular context, there are no specific treatment recommendations for limited metastatic disease. Different treatment approaches are currently used, most of them focusing on local ablative modalities using surgery or radiation [10].

This prospective study aims to describe the clinical and pathologic characteristics of a series of patients with OPCa initially treated with radical prostatectomy. The histological and immunohistochemical features of 27 primary tumors which subsequently developed an oligometastatic status have been evaluated.

\section{Materials and Methods}

This study was conducted under approval of the Cruces University Hospital Ethical Committee (PI2019059) and is compliant with the principles outlined in the Declaration of Helsinki. All patients agreed and signed a consent form.

A total of 27 patients initially treated with radical prostatectomy have been collected at the Department of Radiotherapy Oncology, Cruces University Hospital, Barakaldo, Spain. All patients developed an asymptomatic relapse with $\leq 5$ metachronous metastases after surgery $+/-$ post-operative radiotherapy. The diagnosis and treatment protocol has been described elsewhere [11]. Briefly, oligometastases were diagnosed based on Choline PET/CT scans and were treated with Stereotactic Ablative Radiotherapy (SABR) and/or Volumetric Modulated Arc Therapy (VMAT), and/or Intensity-Modulated Radiation Therapy (IMRT) at our institution between December 2012 and February 2017. Data for these patients were prospectively collected and analyzed.

Following the currently accepted criteria [9], patients were included in the study when they developed up to five metastases during their clinical follow-up, either in the same or different organs. Demographic data and the main clinical findings of all patients were annotated. The indication of metastases directed treatment (MDT) was determined based on the following criteria: a previously treated primary tumor and an apparent complete local response, more than 2 years from diagnosis of the primary tumor to the metastatic recurrence, availability of Ch-PET/CT parameters at positive 
lymph node (LN) or bone metastases, and a maximum of 5 metastatic sites and LN with a diameter of $3 \mathrm{~cm}$ or less.

The nadir plus $2 \mathrm{ng} / \mathrm{mL}$ definition was used to define biochemical relapse, a clinical disease-free survival (cDFS) event was defined as clinical evidence of disease by any clinical, pathological, or radiological method. Finally, the time to castrate-resistant disease was also estimated.

Patients were followed-up with clinical examinations at 1,3, and 6 months in all cases, and thereafter every 1 to 3 months. Prostate-specific antigen (PSA) measurements were scheduled 3-6-monthly during the first year and 6-monthly thereafter. Reassessment with Ch-PET/CT imaging was performed in case of 3 rising PSA values after initial response and a PSA value higher than $2 \mathrm{ng} / \mathrm{mL}$ or if clinically indicated to exclude local or distant metastatic progression. In the case of an oligometastatic recurrence outside the previous SABR/IMRT field, retreatment with SABR/IMRT was offered.

Representative samples of the primary tumor fixed in formalin and embedded in paraffin blocks following routine protocols were analyzed by three pathologists (CM, IZ, JIL) which blindly evaluated the same pathologic criteria: histologic type, Gleason index, 2014 ISUP prognostic grouping [12], UICC/AJCC staging system (8th edition) [13], the presence of intratumor inflammatory cells [quantified as mild (occasional lymphoid aggregates), moderate (one lymphoid aggregate every two high power fields), and intense (one or more lymphoid aggregates per high power field)], and the presence of atrophic changes and stromal reaction in the same histological slides stained with hematoxylin-eosin.

Immunomarkers related with tumor aggressiveness (neurogenesis, proliferative index, neuroendocrine differentiation, and angiogenesis) have been tested in an attempt to delineate, coupled with classical histologic features, any specific profile of this under-recognized clinical presentation of PCa. For such a purpose, Androgen receptor (SP107, Ventana, 760-4605, ready to use, nuclear staining), ERG (EPR3864, Ventana, 790-4576, ready to use, nuclear and cytoplasmic staining), PGP 9.5 (Ventana, 760-4434, ready to use, cytoplasmic staining), Ki67 (30-9, Ventana, 790-4286, ready to use, nuclear staining), chromogranin A (LK2H10, Ventana, 760-2519, ready to use, cytoplasmic staining), synaptophysin (MRQ-40, Ventana, 760-4595, ready to use, cytoplasmic staining), and L1CAM (UJ127.11, Sigma, L4543, dilution 1:100) staining were performed in automated immunostainers (BenchMark Ultra, Ventana Medical Systems) following routine methods. Tris-EDTA was used for antigen retrieval. Negative controls were slides not exposed to the primary antibody, and these were incubated in PBS and then processed under the same conditions as the test slides.

Descriptive statistics were used to summarize patient, tumor, and treatment characteristics. Chi-square and Fisher exact test was used to analyze the relation between histopathological features and clinical outcomes.

\section{Results}

Twenty-seven patients treated with radical prostatectomy and prospectively followed were included in the study. The average follow up was 54 months (range, 26-70). The clinical characteristics are presented in Table 1.

At the time of biochemical relapse after radical prostatectomy, the average age of the patients was 67 years (range, 56-78). Serum PSA levels oscillated between 1.03 and $12.3 \mathrm{ng} / \mathrm{mL}$ (average, $3.41 \mathrm{ng} / \mathrm{mL}$ ). The average PSA doubling time was 4.5 months (range, 1-21). Metastases were located in LN (22/27 cases, $81.5 \%)$ and bones (5/27 cases, $18.5 \%)$.

Local control after the MDT (SABR or VMAT) was successful in 22 cases $(81.5 \%)$. At relapse after initial MDT, 11 out of 27 patients $(40.7 \%)$ developed oligometastatic disease and received subsequent MDT. Of these, 3 patients developed OPCa and were also treated locally with MDT. The treatment in all newly appearing metastases was always the same (SABR or VMAT).

Twenty-two patients (81.5\%) developed biochemical failure after the first MDT. The average of biochemical failure free survival was 21 months (95\%, CI 52.20-57.79). Five patients (18.5\%) developed a local relapse of the treated metastases. The average clinical progression free was 55 months 
(95\%, CI 10.82-31.17). Nine out of 27 (33.3\%) patients developed a castration-resistant status during the follow-up.

Table 1. Clinical data of 27 oligometastatic prostate adenocarcinomas.

\begin{tabular}{ccc}
\hline Clinical Parameters & $n$ & $\%$ \\
\hline Patient age at MDT (mean, range) & $67(56-78)$ & \\
Initial treatment & & 25.9 \\
RP & 7 & 74.1 \\
RP + Salvage RT & 20 & \\
PSA before first MDT (ng/mL) (mean, range) & $3.41(1.03-12.28$ & \\
PSA doubling time in months (mean, range) & $4.5(1-21.5)$ & \\
Follow-up (months) (mean, range) & $54(26-70)$ & 81.5 \\
First oligometastases location & 22 & 18.5 \\
Nodes & 5 & 63 \\
Bones & 17 & 37 \\
Total number of MDTs & 10 & \\
One MDT & & \\
Multiple MDTs &
\end{tabular}

MDT: Metastases directed therapy, RP: Radical prostatectomy, RT: Radiotherapy.

\subsection{Pathological Data}

The results are shown in Table 2. Pathological staging distribution showed 17 (63\%) non-organ confined $(\mathrm{pT3} / \mathrm{b})$ versus $10(37 \%)$ confined $(\mathrm{pT} 2 \mathrm{a} / \mathrm{b} / \mathrm{c})$ cases. Twenty-five cases $(92.5 \%)$ were conventional acinar adenocarcinomas and $2(7.5 \%)$ were duct adenocarcinomas. Intratumor lymphoid infiltrates were mild in 8 cases $(29.6 \%)$, moderate in 10 cases $(37 \%)$, and intense in 9 cases $(33.3 \%)$. Atrophic changes were detected in the adjacent benign prostate tissue in 18 cases $(66.6 \%)$ and tumor stromal fibroblastic reaction in 15 cases (55.5\%). The Gleason score (GS) distribution was as follows: $\mathrm{GS}=6,3$ cases $(11.1 \%) ; \mathrm{GS}=3+4,9$ cases $(33.3 \%) ; \mathrm{GS}=4+3,3$ cases $(11.1 \%)$; and $\mathrm{GS}=8$ to 10 , 12 cases $(44.4 \%)$.

Table 2. Pathological findings in 27 oligometastatic prostate adenocarcinomas.

\begin{tabular}{ccc}
\hline & $n$ & $\%$ \\
\hline pT & & \\
Organ confined (pT2a/b/c) & 9 & 33.3 \\
Non-organ confined (pT3a/b) & 18 & 66.6 \\
Histology & & \\
Acinar & 25 & 92.5 \\
Ductal & 2 & 7.5 \\
Mild & & \\
Inflammatory infiltrates & 8 & 29.6 \\
Moderate & 10 & 37.1 \\
Severe & 9 & 33.3 \\
Atrophy & 18 & 66.6 \\
Stromal reaction & 15 & 55.5 \\
Gleason index & & \\
$3+3$ & 3 & 11.1 \\
$4+3$ & 9 & 33.3 \\
$3+4$ & 3 & 11.1 \\
3 + 5, 4 + 4, $4+5,5+4$ & 12 & 44.4 \\
Prognostic grouping (ISUP 2014) & & \\
1 + 2 & 12 & 44.4 \\
$3+4+5$ & 15 & 55.6 \\
Androgen receptor & 27 & 100 \\
ERG & 11 & 40.7 \\
PGP 9.5 & 20 & 74 \\
Ki-67 (cut-off 5\%) & & \\
$\leq 5$ & 23 & 85.2 \\
$>5$ & 4 & 14.8 \\
Chromogranin A & 8 & 29.6 \\
Synaptophysin & 5 & 18.5 \\
L1CAM & 0 & 0 \\
\hline & &
\end{tabular}


Immunohistochemistry displayed positivity for the androgen receptor in $27 / 27$ cases $(100 \%)$, ERG in $11 / 27$ cases $(40.7 \%)$, PGP 9.5 in $20 / 27$ cases $(74 \%)$, chromogranin A in $8 / 27$ cases $(29.6 \%)$, and synaptophysin in 5/27 cases (18.5\%). Ki67 expression was $\leq 5 \%$ in $23 / 27$ cases (85.1\%). L1CAM was negative in all cases.

\subsection{Statistical Analysis}

The results of the Chi-square (Fisher exact test) are summarized in Table 3. They showed that local control after MDT was associated to non-organ confined tumor category, low Gleason index, stromal reaction, mild inflammation, atrophy, Ki67 > 5\% and $\geq 5 \%$, ERG, PGP 9.5, CRG, and SYN immunostainings. Non-organ confined disease at diagnosis was associated to mild inflammation, Ki-67 index $>5 \%$ and $\geq 5 \%$, chromogranin A and synaptophysin expression. The development of castration-resistant tumors, mild inflammation, atrophy, low Gleason index, CRG, SYN, PGP 9.5, Ki-67 index $>5 \%$ and $\geq 5 \%$ were shown to be dependent variables.

Table 3. Dependent parameters for successful local control, non-organ confined staging, and castration resistance status in 27 oligometastatic prostate adenocarcinomas (Fisher exact test).

\begin{tabular}{lcc}
\hline & & $p$ Value \\
\hline Low inflammation & 0.0003 \\
No atrophy & 0.0008 \\
Nuccessful local control & Organ-confined tumor & 0.0103 \\
& Gleason index 3 + 3 & 0.002 \\
& Gleason index (first pattern 3) & 0.00001 \\
& Ki67 < 5\% & 0.0214 \\
& Ki67 $\leq 5 \%$ & 0.00001 \\
& ERG negative (IHC) & 0.0003 \\
& PGP 9.5 negative (IHC) & 0.0047 \\
& Chromogranin A negative (IHC) & 0.0001 \\
Synaptophysin negative (IHC) & 0.0001 \\
Non-organ confined tumor & High inflammation & 0.00001 \\
\hline Ki67 > 5\% & 0.028 \\
& Ki67 $\geq 5 \%$ & 0.00006 \\
& Chromogranin A positive (IHC) & 0.0281 \\
& Synaptophysin positive (IHC) & 0.01 \\
& High inflammation & 0.002 \\
\hline Castration resistant tumor & Atrophy & 0.006 \\
& Gleason index other than 3+3 & 0.006 \\
& Ki67 > 5\% & 0.00001 \\
& Ki67 $\geq 5 \%$ & 0.0001 \\
& PGP 9.5 positive (IHC) & 0.006 \\
& Synaptophysin positive (IHC) & 0.0024 \\
& & 0.0024 \\
& Chromogranin A positive (IHC) & 0.05 \\
\hline
\end{tabular}

\section{Discussion}

OPCa is a tumor category strictly defined by clinical-radiological criteria $[9,14,15]$. Most series classify as OPCa those cases that develop less than 3-5 metastases during the follow up of the disease [9]. These cases are considered as an intermediate clinical status between localized, organ-confined, and polymetastatic diseases. Either active surveillance [16] or metastasis-directed therapies [17] may be good options to delay systemic treatments in these patients. Hence, the interest generated to identify such cases early at the pre-metastatic phase of the disease. Up to now, however, no distinctive features have been detected at the microscopic level. Oligometastases are usually asymptomatic, so their detection needs close follow up protocols and precise imaging methodologies due to the potential benefit of local therapies in these patients [18]. 
Little is known about the biological reasons for which a tumor may develop either none, few, or many metastases along its evolution. So far, there is no specific histological, immunohistochemical, or molecular feature linked to this clinical behavior in PCa. Metastasis development is a complex stepwise process of adaptation in which tumor cells and the local microenvironment interplay [19]. Speaking in quantitative terms, metastasis is not an efficient process. It has been calculated that only around $0.02 \%$ of circulating tumor cells succeed developing a distant metastasis [20]. For such a purpose, a subset of tumor cells in the primary tumor acquire a specific tropism that includes the secretion of cytokines and growth factors to create the successful local conditions in the target organ for posterior colonization following the Paget's hypothesis [21]. These favorable environmental conditions are site-specific and conform what is called the pre-metastatic niche [22]. Latterly, once arrived in the niche, the metastatic seed needs to initiate immune evasion processes to survive to the local innate immunity transforming macrophages, NK cells, and other elements in allied conspirators. Then arrives an unprecise period of dormancy followed by epigenetic reprogramming processes that will allow $\mathrm{PCa}$ metastatic cells to survive and escape therapies, particularly the androgen receptor signaling blockade, thus converting the neoplasm in a castration-resistant cancer, the common final pathway in the clinical evolution of PCa.

Chronic inflammation, atrophy, and stromal fibroblastic induction [23,24] have been classically associated with prostate cancer initiation and progression. The current findings show that the microbiome associated with chronic inflammation and atrophy allows the development of a specific microenvironment promoting prostate carcinogenesis [24]. Stromal matrix remodeling replaces the prostate fibromuscular stroma and evolves during prostate cancer evolution through transforming growth factor $\beta 1$ regulation [23]. Besides, a very recent study demonstrates that collagen production and accumulation are driven by the activation of PI3K in the prostate epithelium [25]. In this sense, prominent chronic inflammation has been detected in the present series in $33.3 \%$ of the cases, and atrophy and stromal induction in $66.6 \%$ and $55.5 \%$, respectively.

Androgen receptor (AR) blockade evasion is the most common form of therapeutic resistance in PCa and represents an example of tumor adaptation that develops uncontrolled castration-resistant clones [26]. Interestingly, a specific pattern of DNA hypermethylation predicts the development of castration-resistant cancer [27]. All the cases in the present series showed positive immunostaining for AR in the primary tumor but up to $29.6 \%$ of them achieved in the last contact a castration-resistant status.

ERG overexpression promotes the development of androgen independent PCa clones disrupting the androgen receptor [28]. In the clinical practice, the protein tested is generated by the TMPRSS2-ERG fusion genes being positive in a significant percentage of PCa [29]. This series of OPCa expressed this protein in the primary tumor of more than $40 \%$ of the cases.

The importance of neurogenesis in cancer has been very recently reviewed [30]. Axonogenesis identified with PGP 9.5 immunostaining is a predictor of biochemical relapse in the univariate test of PCa and shows a positive correlation with LN status in a series of 640 patients analyzed with computerized digital image analysis [31]. In the present series, up to $74 \%$ of the cases showed evident positive intratumor PGP 9.5 staining.

Several studies have confirmed the positive correlation between Ki-67 high index and bad prognosis in prostate cancer [26,32-34]. This finding is especially interesting in localized prostate cancer, where it is associated with poor disease-specific survival, biochemical failure-free survival, and other parameters of bad prognosis [34]. Also, a high Ki67 index is associated with increased risk of metastases [33] and a bad prognosticator in prostate adenocarcinomas with hormonal blockade [26].

True neuroendocrine differentiation (NED) is rare (0.5-2\%) in PCa before the androgen deprivation therapy is administered but it is significantly increased thereafter (17-30\%) [35]. Several types of NED are associated with a highly aggressive form of $\mathrm{PCa}$, with rapid progression and metastases mainly to visceral organs [36]. After Epstein et al.'s classification [37], it seems that usual prostate adenocarcinomas with focal NED are quite common in the pre-androgen blockade period. Interestingly, this finding does not worsen the prognosis of patients [38]. 
The L1 cellular adhesion molecule (L1CAM) has been implicated in the development of metastases in a wide range of malignant tumors [38]. L1CAM expression has been rarely analyzed in prostate cancer, where it has been associated with androgen-insensitive prostate cancer and high metastatic potential [39]. Since inactivating L1CAM in prostate cancer inhibits metastases [39], this strategy could be an additional potential targeted therapy to be applied in tumors with known aggressive potential. However, the negative immunostaining in the present series suggest that this protein is not an actionable target in OPCa.

To our knowledge, this is the first study looking specifically at pathologic characteristics of OPCa patients. Here, we have found that routine pathological parameters like inflammation, atrophy, Ki-67 index, Gleason index, pathologic staging, and PGP 9.5, chromogranin A, and synaptophysin immunostaining may be relevant in order to achieve better local control rates and to detect castrate resistant disease.

To date, the decision to administrate local ablative treatments in OPCa patients is based only on the clinical characteristics of the patients. Research is being conducted nowadays looking at molecular biomarkers that could help to predict outcomes. Preliminary data suggest that tissue miRNAs may drive metastatic competence by adaptative communication processes between cancer cells and their microenvironment [40]. In this regard, our group has started to investigate the role of the transcriptional co-activator peroxisome proliferator-activated receptor gamma co-activator $1 \alpha$ $(\mathrm{PGC} 1 \alpha)$ in these patients and its association with disease progression.

\section{Conclusions}

OPCa is a category of prostate carcinomas with increasing interest in clinics since the application of local MDT has proven to be very useful in recent years. The initial identification of prostate cancer patients belonging to this category is difficult because of the lack of specific clinical, histological, and immunohistochemical markers. In this study, we have seen that some histological (inflammation and atrophy) and immunohistochemical (Ki-67, PGP 9.5, chromogranin A, and synaptophysin) data are associated with a successful local control of disease and non-organ confined disease, thus helping to make therapeutic decisions. However, further studies are needed to define more precise prognostic identifiers in these patients.

Author Contributions: Conceptualization, A.G.-I. and and J.I.L.; Data curation, A.G.; Formal analysis, C.M., A.G., D.B., A.U., A.E., I.F., R.L., I.Z., R.P., A.C. and A.G.-I.; Investigation, R.P. and A.C.; Methodology, C.M., A.G., D.B., J.G.-O., A.U., A.E., I.F., R.L., I.Z., A.C., A.G.-I. and J.I.L.; Supervision, and J.I.L.; Writing-original draft, C.M., A.G. and R.P.; Writing-review \& editing, A.G.-I. and J.I.L. All authors have read and agreed to the published version of the manuscript.

Funding: This research received no external funding.

Conflicts of Interest: The authors declare no conflict of interest.

\section{References}

1. Siegel, R.L.; Miller, K.D.; Jemal, A. Cancer statistics, 2020. CA Cancer J. Clin. 2020, 70, 3-30. [CrossRef]

2. Hamdy, F.C.; Donovan, J.L.; Lane, J.A.; Mason, M.; Metcalfe, C.; Holding, P.; Davis, M.; Peters, T.J.; Turner, E.L.; Martin, R.M.; et al. 10-Year Outcomes after Monitoring, Surgery, or Radiotherapy for Localized Prostate Cancer. N. Eng. J. Med. 2016, 375, 1415-1424. [CrossRef]

3. Abedi, A.R.; Ghiasy, S.; Fallah-Karkan, M.; Rahavian, A.; Allameh, F. The management of patients diagnosed with incidental prostate cancer. Narrative review. Res. Rep. Urol. 2020, 12, 105-109. [CrossRef]

4. Luzzago, S.; De Cobelli, O.; Cozzi, G.; Peveri, G.; Bagnardi, V.; Catellani, M.; Di Trapani, E.; Mistretta, F.A.; Pricolo, P.; Conti, A.; et al. A novel nomogram to identify candidates for active surveillance amongst patients with International Society of Urological Pathology (ISUP) Grade Group (GG) 1 or ISUP GG2 prostate cancer, according to multiparametric magnetic resonance imaging findings. BJU Int. 2020, 126, 104-113. [CrossRef]

5. Kasivisvanathan, V.; Giganti, F.; Emberton, M.; Moore, C.M. Magnetic resonance imaging should be used in the active surveillance of patients with localised prostate cancer. Eur. Urol. 2020, 77, 318-319. [CrossRef] 
6. Kluytmans, A.; Fütterer, J.J.; Emberton, M.; Sedelaar, M.; Grutters, J. Exploring the risk-reward balance in focal therapy for prostate cancer-a contribution to the debate. Prostate Cancer Prostatic Dis. 2019, 22, 382-384. [CrossRef]

7. Yadav, S.S.; Stockert, J.A.; Hackert, V.; Yadav, K.K.; Tewari, A.K. Intratumor heterogeneity in prostate cancer. Urol. Oncol. 2018, 36, 349-360. [CrossRef]

8. Litwin, M.S.; Tan, H.J. The Diagnosis and Treatment of Prostate Cancer: A Review. JAMA 2017, 317, $2532-2542$. [CrossRef]

9. Tosoian, J.J.; Gorin, M.A.; Ross, A.E.; Pienta, K.J.; Tran, P.T.; Schaeffer, E.M. Oligometastatic prostate cancer: Definitions, clinical outcomes, and treatment considerations. Nat. Rev. Urol. 2017, 14, 15-25. [CrossRef]

10. Gómez-Iturriaga, A.; Ocio, F.C.; Ost, P.; Fernandez, I.; Rodeño, E.; Llarena, R.; Garcia-Olaverri, J.; De Zarate, R.O.; Cacicedo, J.; Ahtamon, A.; et al. Novel insights into the management of oligometastatic prostate cancer: A comprehensive review. Eur. Urol. Oncol. 2019, 2, 174-188. [CrossRef]

11. Gómez-Iturriaga, A.; Casquero Ocio, F.; Ost, P.; Fernández, I.; Rodeño, E.; Llarena, R.; García-Olaverri, J.; Ortiz de Zárate, R.; Cacicedo, J.; Ahtamon, A.; et al. Outcomes after a first and/or second salvage treatment in patients with oligometastatic prostate cancer recurrence detected by (18-F) choline PET-CT. Eur. J. Cancer Care 2019, 28, e13093. [CrossRef]

12. Epstein, J.I.; Egevad, L.; Amin, M.B.; Delahunt, B.; Srigley, J.R.; Humphrey, P.A. The 2014 International Society of Urological Pathology (ISUP) Consensus Conference on Gleason grading of prostatic carcinoma: Definition of grading patterns and proposal for a new grading system. Am. J. Surg. Pathol. 2016, 40, $244-252$. [CrossRef]

13. Paner, G.P.; Stadler, W.M.; Hansel, D.E.; Montironi, R.; Lin, D.W.; Amin, M.B. Updates in the Eighth Edition of the Tumor-Node-Metastasis Staging Classification for urologic cancers. Eur. Urol. 2018, 73, 560-569. [CrossRef]

14. Paner, G.P.; Stadler, W.M.; Hansel, D.E.; Montironi, R.; Lin, D.W.; Amin, M.B. Oligometastatic prostate cancer: The game is afoot. Cancer Treat. Rev. 2019, 73, 84-90. [CrossRef]

15. Foster, C.C.; Weichselbaum, R.R.; Pitroda, S.P. Oligometastatic prostate cancer. Reality or figment of imagination. Cancer 2019, 125, 340-352. [CrossRef]

16. Ost, P.; Reynders, D.; Decaestecker, K.; Fonteyne, V.; Lumen, N.; De Bruycker, A.; Lambert, B.; Delrue, L.; Bultijnck, R.; Claeys, T.; et al. Surveillance or metastasis-directed therapy for oligometastatic prostate cancer recurrence. A prospective, randomized, multicentre phase II trial. J. Clin. Oncol. 2018, 36, 446-453. [CrossRef]

17. Lecouvet, F.E.; E Oprea-Lager, D.; Liu, Y.; Ost, P.; Bidaut, L.; Collette, L.; Deroose, C.M.; Goffin, K.; Herrmann, K.; Hoekstra, O.S.; et al. Use of modern imaging methods to facilitate trials of metastasis-directed therapy for oligometastatic disease in prostate cancer: A consensus recommendation from the EORTC Imaging Group. Lancet Oncol. 2018, 19, e534-e545. [CrossRef]

18. Franklin, J.M.; Sharma, R.A.; Harris, A.L.; Gleeson, F.V. Imaging oligometastatic cancer before local treatment. Lancet Oncol. 2016, 17, e406-e414. [CrossRef]

19. Chambers, A.F.; Groom, A.C.; MacDonald, I.C. Dissemination and growth of cancer cells in metastatic sites. Nat. Rev. Cancer 2002, 2, 563-572. [CrossRef]

20. Tharp, D.; Nandana, S. How prostate cancer cells use strategy instead of brute force to achieve metastasis. Cancers 2019, 11, 1928. [CrossRef]

21. Paget, S. The distribution of secondary growths in cancer of the breast. Cancer Metastasis Rev. 1989, 8, 98-101. [CrossRef]

22. Kaplan, R.N.; Riba, R.D.; Zacharoulis, S.; Bramley, A.H.; Vincent, L.; Costa, C.; Macdonald, D.D.; Jin, D.K.; Shido, K.; Kerns, S.A.; et al. VEGFR1-positive hematopoietic bone marrow progenitors initiate the pre-metastatic niche. Nature 2005, 438, 820-827. [CrossRef] [PubMed]

23. Tuxhorn, J.A.; Ayala, G.E.; Smith, M.J.; Smith, V.C.; Dang, T.D.; Rowley, D.R. Reactive stroma in human prostate cancer: Induction of myofibroblasts phenotype and extracellular matrix remodeling. Clin. Cancer Res. 2002, 8, 2912-2923. [PubMed]

24. Sfanos, K.S.; Yegnasubramanian, S.; Nelson, W.G.; de Marzo, A.M. The inflammatory microenvironment and microbiome in prostate cancer development. Nat. Rev. Urol. 2018, 15, 11-24. [CrossRef] 
25. Wegner, K.A.; Mueller, B.R.; Unterberger, C.J.; Avila, E.J.; Ruetten, H.; Turco, A.E.; Oakes, S.R.; Girardi, N.M.; Halberg, R.B.; Swanson, S.M.; et al. Prostate epithelial-specific expression of the activated PI3K drives stromal collagen production and accumulation. J. Pathol. 2020, 250, 231-242. [CrossRef]

26. Angulo, J.C.; Redondo, C.; Sánchez-Chapado, M.; Colás, B.; Ropero, S.; López, J.I. Survival predictors in patients with prostate adenocarcinoma with hormonal blockade. Pathol. Res. Pract. 2016, 212, 899-903. [CrossRef]

27. Angulo, J.C.; Andrés, G.; Ashour, N.; Sánchez-Chapado, M.; López, J.I.; Ropero, S. Development of castration resistant prostate cancer can be predicted by a DNA hypermethylation profile. J. Urol. 2016, 195, 619-626. [CrossRef]

28. Yu, J.; Yu, J.; Mani, R.-S.; Cao, Q.; Brenner, C.J.; Cao, X.; Wang, G.X.; Wu, L.; Li, J.; Hu, M.; et al. An integrated network of androgen receptor, polycomb, and TMPRSS2-ERG gene fusions in prostate cancer progression. Cancer Cell 2010, 17, 443-454. [CrossRef]

29. Clark, J.; Merson, S.; Jhavar, S.; Flohr, P.; Edwards, S.; Foster, C.S.; Eeles, R.; Martin, F.L.; Phillips, D.H.; Crundwell, M.; et al. Diversity of TMPRSS2-ERG fusion transcripts in the human prostate. Oncogene 2007, 26, 2667-2673. [CrossRef]

30. Faulkner, S.; Jobling, P.; March, B.; Jiang, C.C.; Hondermarck, H. Tumor neurobiology and the war of nerves in cancer. Cancer Discov. 2019, 9, 702-710. [CrossRef]

31. Olar, A.; He, D.; Florentin, D.; Ding, Y.; Ayala, G. Biologic correlates and significance of axonogenesis in prostate cancer. Hum. Pathol. 2014, 45, 1358-1364. [CrossRef] [PubMed]

32. Fisher, G.J.; on behalf of the Transatlantic Prostate Group; Yang, Z.H.; Kudahetti, S.C.; Moller, H.; Scardino, P.T.; Cuzick, J.; Berney, D. Prognostic value of Ki-67 for prostate cancer death in a conservatively managed cohort. Br. J. Cancer 2013, 108, 271-277. [CrossRef] [PubMed]

33. Green, W.J.; Ball, G.; Hulman, G.; Johnson, C.; Van Schalwyk, G.; Ratan, H.L.; Soria, D.; Garibaldi, J.M.; Parkinson, R.; Hulman, J.; et al. Ki67 and DLX2 predict increased risk of metastasis formation in prostate cancer. A targeted molecular approach. Br. J. Cancer 2016, 115, 236-242. [CrossRef] [PubMed]

34. Berlin, A.; Castro-Mesta, J.F.; Rodriguez-Romo, L.; Hernandez-Barajas, D.; González-Guerrero, J.F.; Rodríguez-Fernández, I.A.; González-Conchas, G.; Verdines-Perez, A.; Vera-Badillo, F.E. Prognostic role of Ki-67 in localized prostate cancer. A systematic review and meta-analysis. Urol. Oncol. 2017, 35, 499-506. [CrossRef]

35. Patel, G.K.; Chugh, N.; Tripathy, M. Neuroendocrine differentiation of prostate cancer. An intriguing example of tumor evolution at play. Cancers 2019, 11, 1405. [CrossRef]

36. Vlachostergios, P.J.; Puca, L.; Beltran, H. Emerging variants of castration-resistant prostate cancer. Curr. Oncol. Rep. 2017, 19, 32. [CrossRef]

37. Epstein, J.I.; Amin, M.B.; Beltran, H.; Lotan, T.L.; Mosquera, J.-M.; Reuter, V.E.; Robinson, B.D.; Troncoso, P.; Rubin, M.A. Proposed morphologic classification of prostate cancer with neuroendocrine differentiation. Am. J. Surg. Pathol. 2014, 38, 756-767. [CrossRef]

38. Altevogt, P.; Doberstein, K.; Fogel, M. L1CAM in human cancer. Int. J. Cancer 2015, 138, 1565-1576. [CrossRef]

39. Sung, S.Y.; Wu, I.H.; Chuang, P.H.; Petros, J.A.; Wu, H.C.; Zeng, H.J.; Huang, W.C.; Chung, L.W.K.; Hsieh, C.L. Targeting L1 cell adhesion molecule expression using liposome-encapsulated siRNA suppresses prostate cancer bone metastasis and growth. Oncotarget 2014, 5, 991-9929. [CrossRef]

40. Ferrer Albiach, C.; Ochoa Aranda, E.; Gómez-Iturriaga, A.; Sotoca Ruiz, A.; López Campos, F.; Porras Martinez, F.; García Gómez, R.; Algara López, M.; Ramos Fernández, V.; Conde Moreno, A.; et al. MicroRNA95 may be involved in oligometastatic prostate cancer. J. Cancer Treat. Res. 2019, 7, 33-40. [CrossRef]

Publisher's Note: MDPI stays neutral with regard to jurisdictional claims in published maps and institutional affiliations. 